\title{
Liquid-glass transition of confined fluids: Some insights from a mode-coupling theory
}

\author{
V Krakoviack \\ Laboratoire de Chimie, UMR CNRS 5182, École Normale Supérieure de Lyon, 46, \\ Allée d'Italie, 69364 Lyon Cedex 07, France \\ E-mail: vincent.krakoviack@ens-lyon.fr
}

\begin{abstract}
The dynamics of confined glassforming liquids is discussed on the basis of the recent extension of the mode coupling theory for the liquid-glass transition to the model of the quenched-annealed binary mixture. It is in particular shown that, in confinement, the density correlation functions always decay to a non-zero infinite time value, even in the fluid state, and some clarification is given about the question of the relation between structure and dynamics in confined fluids.

PACS numbers: 64.70.Pf, 61.20.Lc, 61.25.-f
\end{abstract}

In the past few years, there has been a strong interest for the dynamics of liquids under confinement and more specifically for their structural glass transition, which has been investigated by a variety of experimental techniques and by computer simulation [1, 2]. One of the main goals of these studies was to improve our understanding of the concept of cooperativity, a key ingredient of many glass transition theories [3]. Indeed, there are now many evidences that, in the bulk, the dynamics of deeply supercooled liquids is strongly inhomogeneous and that correlated clusters of molecules, the so-called dynamical heterogeneities, play a crucial role in the slowing-down of the dynamics when the temperature is decreased. But, up to now, many aspects of the characterization of these dynamical heterogeneities have remained elusive. For instance, there is no clear consensus on their shape, their size and their evolutions with temperature.

This is where the interest for confined glassforming systems came in. Indeed, confinement is a means to introduce geometrical constraints and new characteristic lengthscales (pore size, film thickness...) in the system under study. Thus, by looking at the way the dynamics is modified under confinement compared to the bulk, one can hope to gain some insight on the properties of the dynamical heterogeneities. For instance, in the simplest scenarios, one expects from finite size effects a cutoff on the slowing-down of the dynamics as temperature is varied, when the typical size of the heterogeneities in the bulk would become larger than the characteristic lengthscale of the confining medium. Confinement would thus provide an indirect probe of the properties of the dynamical heterogeneities. 
It turns out that the situation is more complex. Indeed, the previous line of reasoning requires that the physical phenomena which are specific of confined systems have a negligible impact on the dynamics of the imbibed fluid or at least that their influence is sufficiently well known that it can subtracted from the results. This is usually not the case and strong confinement effects are observed, in particular at the fluid-solid interface where structured layers of almost immobile molecules are often formed. So this is in fact the problem of dynamics under confinement as a whole which has to be addressed and not only at the level of a simple modification of the bulk dynamics.

Theories dealing with the dynamics in confinement are far less developed than for the bulk and they usually are of a phenomenological nature [2]. There is yet a strong need for elaborate microscopic theories in this field. Indeed, the variety of systems to consider is immense. Porous media can differ in the shape, size, size distribution, connectivity, etc, of their pores. They can be made of various materials, leading to a wide range of fluid-solid interactions which adds to the already great variability of intermolecular interactions met with usual glassformers. A reasonable theoretical approach, able to catch many of these subtleties, would thus be of a great help. Applied to various models, it would allow to explore thoroughly the phenomenology of confined glassforming systems and maybe to disentangle the different physical effects which interplay in these systems.

Recently, a step in this direction has been made with the extension of the mode coupling theory (MCT) for the liquid-glass transition [4, 5, 6, 7] to a particular class of confined systems, the so-called "quenched-annealed" (QA) binary mixtures [8]. In these systems, first introduced by Madden and Glandt [9], the fluid molecules equilibrate in a matrix of particles frozen in a disordered configuration sampled from a given probability distribution. This class of models, which describe situations of statistically homogeneous and isotropic confinement, is thought to be able to reproduce most of the physics of fluids confined in materials like Vycor, controlled porous glasses or aerogels, and, in fact, the fluid dynamics and glass transition in some of its instances have been the subject of recent studies by molecular dynamics simulations [10, 11, 12.

In this paper, we will give a short presentation of the proposed extension of the MCT to QA systems (a more detailed account is given in reference [8]) and, on the basis of this approach, we will discuss a few aspects of the physics of confined fluids which are of relevance for the interpretation of experimental and computer simulation results.

Before dealing with dynamics, one first has to consider some peculiarities of the statics of QA systems. QA mixtures are systems with quenched disorder, so that their theoretical description requires two types of averages, a thermal average denoted by $\langle\cdots\rangle$, taken for a given realization of the matrix, and a disorder average over the matrix realizations, denoted by $\cdots$, to be taken after the thermal average. Like in the bulk, one is interested in the Fourier components of the microscopic fluid density, or, in short, density fluctuations, defined as $\rho_{\mathbf{q}}^{f}(t)=\sum_{j=1}^{N_{f}} e^{i \mathbf{q r}_{j}(t)}$, where $\mathbf{q}$ denotes the wavevector, $N_{f}$ is the fluid particle number and $\mathbf{r}_{j}(t)$ is the position of fluid particle $j$ at time $t$. A significant difference with the bulk is that, for a given matrix 
realization, the translational invariance of the system is broken by the presence of the quenched component. This results in non-zero average density fluctuations at equilibrium, i.e., $\left\langle\rho_{\mathbf{q}}^{f}\right\rangle \neq 0$. It is only after the disorder average that the symmetry is restored, leading to $\overline{\left\langle\rho_{\mathbf{q}}^{f}\right\rangle}=0$, hence the description of the model as statistically homogeneous and isotropic. This property has a well known impact on the equations describing the structural correlations in such systems, for instance the so-called replica Ornstein-Zernike (OZ) equations [13, 14, where it leads to the splitting of the total and direct correlation functions of the fluid, $h^{f f}(r)$ and $c^{f f}(r)$, respectively, into two contributions, connected $\left[h^{c}(r)\right.$ and $\left.c^{c}(r)\right]$ and blocked or disconnected $\left[h^{b}(r)\right.$ and $\left.c^{b}(r)\right]$. The separation of $h^{f f}(r)$ into two terms leads to a similar property of the fluid structure factor $S_{q}^{f f}=\overline{\left\langle\rho_{\mathbf{q}}^{f} \rho_{-\mathbf{q}}^{f}\right\rangle} / N_{f}=1+n_{f} \hat{h}_{q}^{f f}$, where $n_{f}$ is the fluid number density, which can be expressed as $S_{q}^{f f}=S_{q}^{c}+S_{q}^{b}$ with $S_{q}^{c}=\overline{\left\langle\left(\rho_{\mathbf{q}}^{f}-\left\langle\rho_{\mathbf{q}}^{f}\right\rangle\right)\left(\rho_{-\mathbf{q}}^{f}-\left\langle\rho_{-\mathbf{q}}^{f}\right\rangle\right)\right\rangle} / N_{f}=1+n_{f} \hat{h}_{q}^{c}$ and $S_{q}^{b}=\overline{\left\langle\rho_{\mathbf{q}}^{f}\right\rangle\left\langle\rho_{-\mathbf{q}}^{f}\right\rangle} / N_{f}=n_{f} \hat{h}_{q}^{b}$, where $\hat{f}_{q}$ denotes the Fourier transform of $f(r)$. To fix all the notations, we define here the matrix-matrix and fluid-matrix structure factors and total correlation functions as well, which are given by $S_{q}^{m m}=\overline{\left\langle\rho_{\mathbf{q}}^{m} \rho_{-\mathbf{q}}^{m}\right\rangle} / N_{m}=1+n_{m} \hat{h}_{q}^{m m}$ and $S_{q}^{f m}=\overline{\left\langle\rho_{\mathbf{q}}^{f} \rho_{-\mathbf{q}}^{m}\right\rangle} / \sqrt{N_{f} N_{m}}=\sqrt{n_{f} n_{m}} \hat{h}_{q}^{f m}$, where $N_{m}$ is the matrix particle number, $n_{m}$ is the matrix number density, and $\rho_{\mathbf{q}}^{m}=\sum_{j=1}^{N_{m}} e^{i \mathbf{q s}_{j}}$, where $\mathbf{s}_{j}$ is the fixed position of matrix particle $j$, is the $\mathbf{q}$ Fourier component of the quenched microscopic matrix density.

The identification of two types of static fluid correlations has significant implications for the dynamics. Indeed, if one forgets for a moment the possibility of a dynamical ergodicity breaking, one expects, using standard arguments, that $\lim _{t \rightarrow \infty} \overline{\left\langle\rho_{\mathbf{q}}^{f}(t) \rho_{-\mathbf{q}}^{f}\right\rangle}=$ $\overline{\left\langle\rho_{\mathbf{q}}^{f}\right\rangle\left\langle\rho_{-\mathbf{q}}^{f}\right\rangle}$, i.e., the normalized total density fluctuation autocorrelation function $\phi_{q}^{T}(t)=$ $\overline{\left\langle\rho_{\mathbf{q}}^{f}(t) \rho_{-\mathbf{q}}^{f}\right\rangle} /\left(N_{f} S_{q}^{f f}\right)$ does not decay to zero at long times, but rather

$$
\lim _{t \rightarrow \infty} \phi_{q}^{T}(t)=\frac{S_{q}^{b}}{S_{q}^{f f}} .
$$

This general result only derives from the fact that the fluid is plunged in an inhomogeneous external potential, which here is due to the matrix. The existence of a non-zero limit thus is independent of the model and in particular it does not depend on the fluid-matrix interaction. To use the language of scattering experiments, it means that one should always expect to measure non-vanishing intermediate scattering functions or that an elastic contribution has to be present in the dynamical structure factors [15, 16. We stress here that this is a true static phenomenon, and not one related to some ultra slow dynamical process.

We can now turn to the dynamical theory. Following the preceding discussion, the proper dynamical variable to consider is the relaxing part of the fluid density fluctuations $\delta \rho_{\mathbf{q}}^{f}(t)=\rho_{\mathbf{q}}^{f}(t)-\left\langle\rho_{\mathbf{q}}^{f}\right\rangle$, rather than the full $\rho_{\mathbf{q}}^{f}(t)$. Then, using standard projection operator methods [5], the equations of the MCT for QA systems are obtained [8]. They are equations for the time evolution of the normalized connected density fluctuation autocorrelation function, $\phi_{q}(t)=\overline{\left\langle\delta \rho_{\mathbf{q}}^{f}(t) \delta \rho_{-\mathbf{q}}^{f}\right\rangle} /\left(N_{f} S_{q}^{c}\right)$, and they consist of a generalized 
Langevin equation, which is the same as for the bulk,

$$
\ddot{\phi}_{q}+\Omega_{q}^{2} \phi_{q}+\Omega_{q}^{2} \int_{0}^{t} d \tau M_{q}(t-\tau) \dot{\phi}_{q}(\tau)=0,
$$

with $\Omega_{q}^{2}=q^{2} k_{B} T /\left(m S_{q}^{c}\right)$, where $m$ is the mass of the fluid particles, $T$ the temperature and $k_{B}$ the Boltzmann constant, and of an expression for the memory kernel $M_{q}(t)=$ $\Gamma_{q} \delta(t)+M_{q}^{(\mathrm{MC})}(t)$, with

$$
\begin{aligned}
& M_{q}^{(\mathrm{MC})}(t)=\int \frac{d^{3} \mathbf{k}}{(2 \pi)^{3}}\left[V_{\mathbf{q}, \mathbf{k}}^{(2)} \phi_{k}(t) \phi_{|\mathbf{q}-\mathbf{k}|}(t)+V_{\mathbf{q}, \mathbf{k}}^{(1)} \phi_{k}(t)\right], \\
& V_{\mathbf{q}, \mathbf{k}}^{(2)}=\frac{1}{2} n_{f} S_{q}^{c}\left[\frac{\mathbf{q} \cdot \mathbf{k}}{q^{2}} \hat{c}_{k}^{c}+\frac{\mathbf{q} \cdot(\mathbf{q}-\mathbf{k})}{q^{2}} \hat{c}_{|\mathbf{q}-\mathbf{k}|}^{c}\right]^{2} S_{k}^{c} S_{|\mathbf{q}-\mathbf{k}|}^{c}, \\
& V_{\mathbf{q}, \mathbf{k}}^{(1)}=n_{m} S_{q}^{c}\left[\frac{\mathbf{q} \cdot(\mathbf{q}-\mathbf{k})}{q^{2}}+n_{f} \frac{\mathbf{q} \cdot \mathbf{k}}{q^{2}} \hat{c}_{k}^{c}\right]^{2} \frac{\left(\hat{h}_{|\mathbf{q}-\mathbf{k}|}^{f m}\right)^{2}}{S_{|\mathbf{q}-\mathbf{k}|}^{m m}} S_{k}^{c} .
\end{aligned}
$$

There are strong analogies between these equations and those for the bulk. Indeed, they share the same mathematical structure, so that most of their properties are already known from the extensive study of reference [5]. At a more detailed level, the expressions of the vertices $V_{\mathbf{q}, \mathbf{k}}^{(2)}$ and $V_{\mathbf{q}, \mathbf{k}}^{(1)}$, which determine the long time behaviour of $\phi_{q}(t)$ and involve static quantities only, are very similar to those found in the bulk, for collective and tagged particle density relaxations, respectively [4.

About these vertices, a specific point is worth stressing. Indeed, one can see that the structural quantities on which they depend are not those related to the full density fluctuations, but the connected ones, which specifically characterize the static correlations of the relaxing part of the density fluctuations. This result, whose generality, we believe, goes beyond the model of QA mixtures, solves an apparent paradox mentioned in various places and sometimes considered as a challenge to a mode coupling description of the glass transition in confinement, that systems with identical structural properties can have significantly different dynamical behaviours [11, 17]: In fact, only a nontrivial fraction of the total density fluctuations, a fraction which exists on top of the static average density fluctuations induced by the external potential in which the fluid particles evolve, is "active" in the determination of the dynamical properties of the confined fluid, and it is the evolution of this contribution which has to be considered to discuss the relation between the fluid structure and dynamics and not the total structure functions of the system.

The solution of the MCT equations for QA mixtures offers no special difficulties compared to the bulk, since we essentially deal with the same type of equations. In reference [8], we have obtained by the methods of reference [18] the dynamical phase diagram of a simple system consisting of a fluid of hard spheres confined in a matrix of hard spheres frozen in an equilibrium configuration, with both types of particles having diameter $\sigma$ [12. We present here typical examples of the time evolution of the predicted density correlation functions. Like in previous work, the system is characterized by two volume fractions $\phi_{f}=\pi n_{f} \sigma^{3} / 6$ and $\phi_{m}=\pi n_{m} \sigma^{3} / 6$, and the necessary structural 

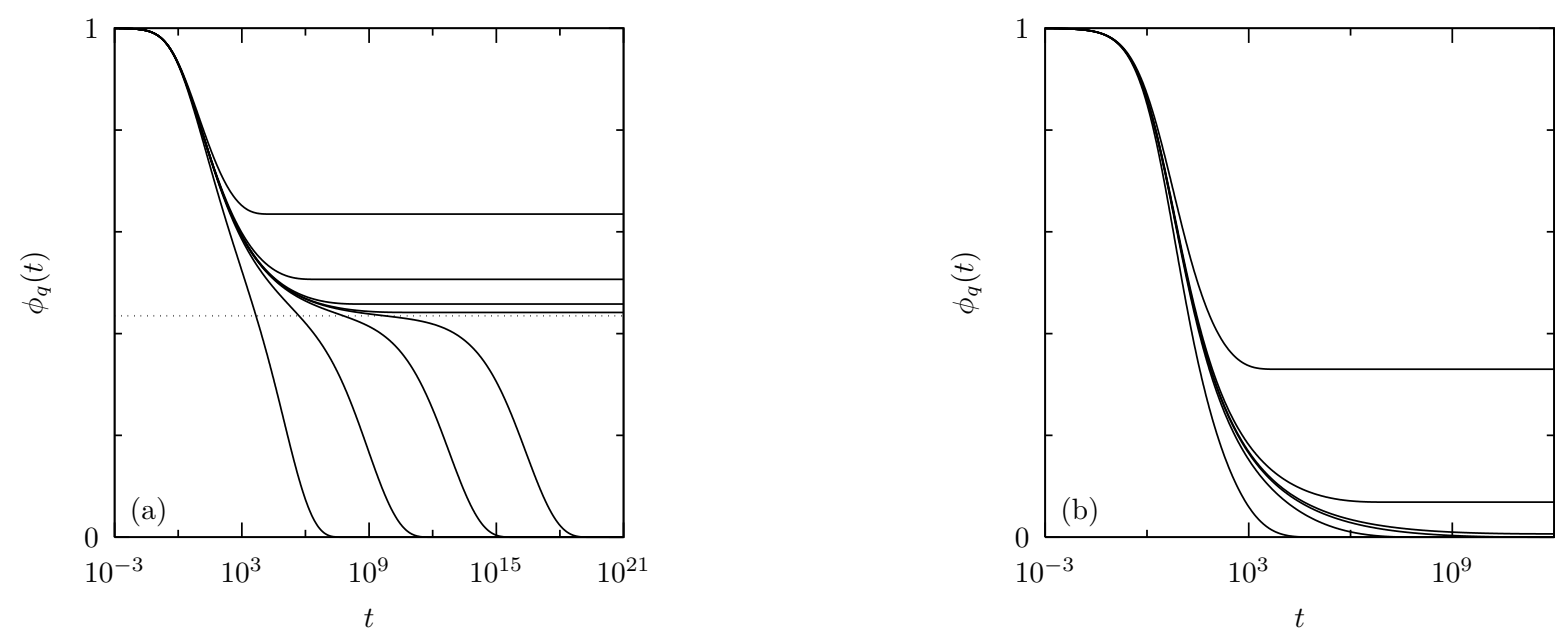

Figure 1. Connected density correlation function $\phi_{q}(t)$ for hard sphere fluids confined in matrices of identical hard spheres frozen in an equilibrium configuration. $q \sigma \simeq 6.71$ is close to the position of the main maximum of the fluid structure factor. We use $t_{\text {mic }}=160$. (a) $\phi_{m}=0.2$; from bottom to top: $\phi_{f}=0.99 \phi_{f}^{c}, 0.999 \phi_{f}^{c}, 0.9999 \phi_{f}^{c}$, $0.99999 \phi_{f}^{c}, 1.00001 \phi_{f}^{c}, 1.0001 \phi_{f}^{c}, 1.001 \phi_{f}^{c}, 1.01 \phi_{f}^{c}$, where $\phi_{f}^{c} \simeq 0.672$ is the critical fluid compacity. The dotted line shows the asymptote of $\phi_{q}(t)$ at $\phi_{f}^{c}$. (b) $\phi_{m}=0.4$; from bottom to top: $\phi_{f}=0.9 \phi_{f}^{c}, 0.99 \phi_{f}^{c}, 0.999 \phi_{f}^{c}, 1.001 \phi_{f}^{c}, 1.01 \phi_{f}^{c}, 1.1 \phi_{f}^{c}$, where $\phi_{f}^{c} \simeq 0.279$ is the critical compacity.

quantities are computed using the Percus-Yevick approximation [13, 19]. For simplicity, we neglect $\ddot{\phi}_{q}(t)$ in the generalized Langevin equation, so that the transient dynamics of $\phi_{q}(t)$ now has a time constant $\tau_{q}=t_{\text {mic }} S_{q}^{c} /(q \sigma)^{2}[18$.

Two types of ergodicity breaking transitions were found in reference [8]. For small matrix densities, a type B or discontinuous ideal glass transition line is met. It corresponds to the well known scenario found in the bulk, showing a two-step relaxation in the ergodic phase and a discontinuous change of the asymptotic value of $\phi_{q}(t)$ from zero to a finite value when the transition line is reached, corresponding to the divergence of the characteristic time of the second relaxation step. This is illustrated in figure 1a. For larger matrix densities, a type $\mathrm{A}$ or continuous ideal glass transition line is found. Here, one observes a one-step relaxation and the ergodicity breaking transition is continuous, i.e., the non-zero asymptotic value of $\phi_{q}(t)$ grows continuously from zero when the system enters in the non-ergodic domain. Such a behaviour is shown in figure $1 \mathrm{~b}$.

From the knowledge of $\phi_{q}(t)$, one can compute the total density correlation function $\phi_{q}^{T}(t)$, which is the quantity directly measurable in experiments and simulations. Both functions are indeed related by the simple formula

$$
\phi_{q}^{T}(t)=\frac{S_{q}^{c}}{S_{q}^{f f}} \phi_{q}(t)+\frac{S_{q}^{b}}{S_{q}^{f f}} .
$$

In figure 2 are reported the results of this transformation on the data of figure 1. From these curves, difficulties can immediately be anticipated if one is to compare the predictions of the mode coupling theory with experimental or simulation data for 

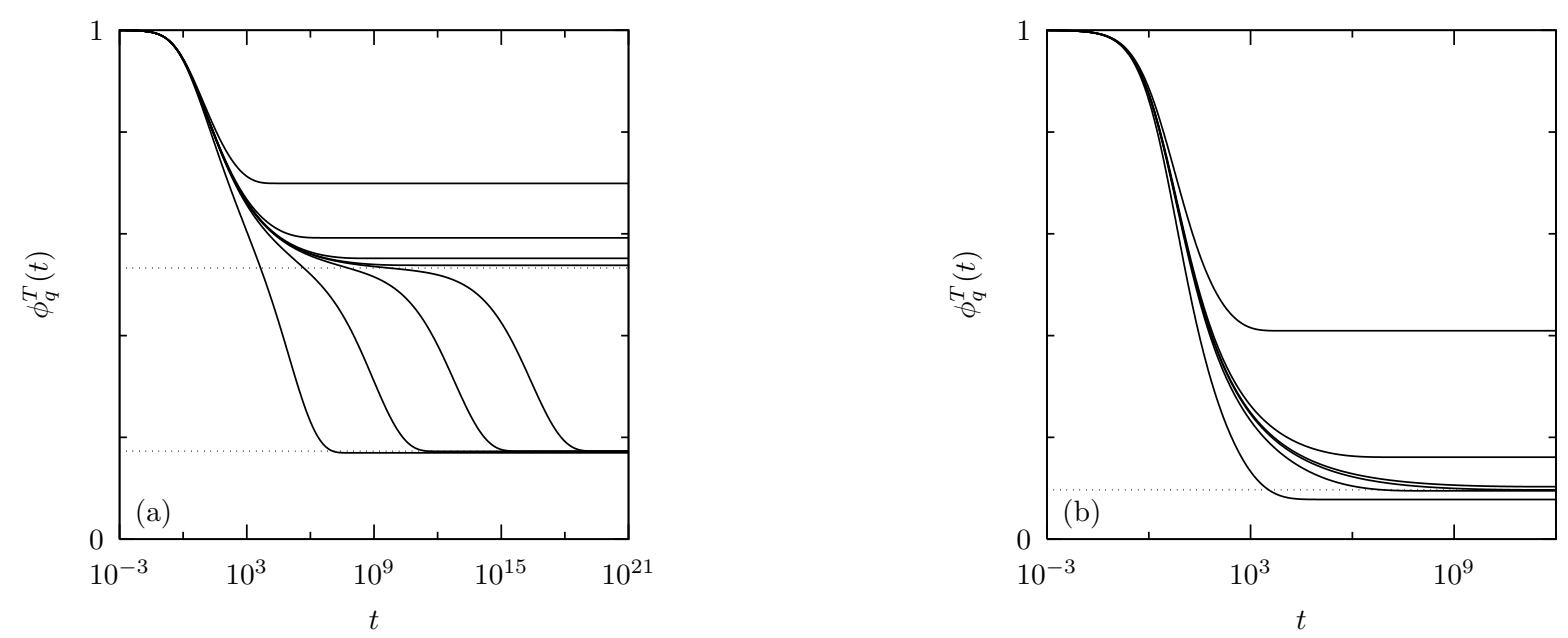

Figure 2. Total density correlation function $\phi_{q}^{T}(t)$ for the same systems as in figure 1. (a) $\phi_{m}=0.2 ; \phi_{f}$ 's as in figure 1a. The lowest dotted line shows the ratio $S_{q}^{b} / S_{q}^{f f}$ at $\phi_{f}^{c}$, while the highest shows the corresponding asymptote of $\phi_{q}^{T}(t)$. (b) $\phi_{m}=0.4$; $\phi_{f}$ 's as in figure $1 \mathrm{~b}$. The dotted line shows the ratio $S_{q}^{b} / S_{q}^{f f}$ at $\phi_{f}^{c}$.

$\phi_{q}^{T}(t)$. Indeed, one sees that the blocked part of the static density correlations provides a density dependent background on top of which the glassy dynamics develops itself. It might thus be difficult to separate, in the long time behaviour of $\phi_{q}^{T}(t)$, the evolutions which are of purely static origin from those which characterize the glassy dynamics of the system. This is especially critical in the case of type A transitions, since when the system enters in the ideal glassy state both contributions are, to first order, linear in $\phi_{f}-\phi_{f}^{c}[5]$.

In conclusion, we have demonstrated how the model of the QA mixture can be a very useful tool for a better understanding of the physics of confined glassforming liquids. Indeed, we have shown that this model, thanks to some crucial simplifying features, in particular the property of statistical homogeneity, allows for a rigorous illustration of some essential concepts which have to be taken into account when dealing with confined systems, like the distinction between static and relaxing density fluctuations. Now, with the corresponding extension of the MCT which is available, it seems that much progress could be done in our understanding of the dynamics of confined fluid systems.

\section{References}

[1] See for instance Proceedings of the International Workshop on Dynamics in Confinement $2000 \mathrm{~J}$. Phys. (Paris) IV 10 Pr7-203 and Proceedings of Second International Workshop on Dynamics in Confinement 2003 Eur. Phys. J. E 12 3-204

[2] For a recent review see Alcoutlabi M and McKenna G B 2005 J. Phys.: Condens. Matter 17 R461

[3] Sillescu H 1999 J. Non-Cryst. Solids 24381

Ediger M D 2000 Annu. Rev. Phys. Chem. 5199

Richert R 2002 J. Phys.: Condens. Matter 14 R703

[4] Bengtzelius U, Götze W and Sjölander A 1984 J. Phys. C 175915 
[5] Götze W 1991 Liquids, freezing and glass transition edited by Hansen J-P, Levesque D and ZinnJustin J (Amsterdam: North Holland) p 287

[6] Götze W and Sjögren L 1992 Rep. Prog. Phys. 55241

[7] Götze W 1999 J. Phys.: Condens. Matter 11 A1

[8] Krakoviack V 2005 Phys. Rev. Lett. 94065703

[9] Madden W G and Glandt E D 1988 J. Stat. Phys. 51537

Madden W G 1992 J. Chem. Phys. 965422

[10] Gallo P, Pellarin R and Rovere M 2002 Europhys. Lett. 57212

Gallo P, Pellarin R and Rovere M 2003 Phys. Rev. E 67041202

Gallo P, Pellarin R and Rovere M 2003 Phys. Rev. E 68061209

[11] Kim K 2003 Europhys. Lett. 61790

[12] Chang R, Jagannathan K and Yethiraj A 2004 Phys. Rev. E 69051101

[13] Given J A and Stell G 1992 J. Chem. Phys. 974573

Lomba E, Given J A, Stell G, Weis J J and Levesque D 1993 Phys. Rev. E 48233

Given J A and Stell G 1994 Physica A 209495

[14] Rosinberg M L, Tarjus G and Stell G 1994 J. Chem. Phys. 1005172

[15] Zorn R, Hartmann L, Frick B, Richter D and Kremer F 2002 J. Non-Cryst. Solids 307547

[16] Alba-Simionesco C, Dosseh G, Dumont E, Frick B, Geil B, Morineau D, Teboul V and Xia Y 2003 Eur. Phys. J. E 1219

[17] Scheidler P, Kob W and Binder K 2004 J. Phys. Chem. B 1086673

[18] Franosch T, Fuchs M, Götze W, Mayr M R and Singh AP 1997 Phys. Rev. E 557153

[19] Meroni A, Levesque D and Weis J J 1996 J. Chem. Phys. 1051101 